\title{
Modelling and Analysis of Complex Viscous Fluid in Thin Elastic Tubes
}

\author{
Yufang Gao' and Zongguo Zhang $\mathbb{D}^{2}$ \\ ${ }^{1}$ College of Applied Meteorology, Jiangsu Key Laboratory of Agricultural Meteorology, \\ Nanjing University of Information Science and Technology, 219 Ning Liu Street, Nanjing 210044, China \\ ${ }^{2}$ School of Mathematics and Statistics, Qilu University of Technology (Shandong Academy of Sciences), Jinan 250353, China
}

Correspondence should be addressed to Zongguo Zhang; zhangzongguo@qlu.edu.cn

Received 20 July 2020; Revised 19 August 2020; Accepted 27 August 2020; Published 15 September 2020

Academic Editor: Tongqian Zhang

Copyright (c) 2020 Yufang Gao and Zongguo Zhang. This is an open access article distributed under the Creative Commons Attribution License, which permits unrestricted use, distribution, and reproduction in any medium, provided the original work is properly cited.

\begin{abstract}
Cardiovascular disease is a major threat to human health. The study on the pathogenesis and prevention of cardiovascular disease has received special attention. In this paper, we have contributed to the derivation of a mathematical model for the nonlinear waves in an artery. From the Navier-Stokes equations and continuity equation, the vorticity equation satisfied by the blood flow is established. And based on the multiscale analysis and perturbation method, a new model of the Boussinesq equation with viscous term is derived to describe the propagation of a viscous fluid through a thin tube. In order to be more consistent with the flow of the fluid, the time-fractional Boussinesq equation with viscous term is deduced by employing the semi-inverse method and the fractional variational principle. Moreover, the approximate analytical solution of the fractional equation is obtained, and the effect of viscosity on the amplitude and width of the wave is studied. Finally, the effects of the fractional order parameters and vessel radius on blood flow volume are discussed and analyzed.
\end{abstract}

\section{Introduction}

In recent years, in the field of biological rheology, the rheology which is related to the blood, blood vessels, and heart that constitute the human blood circulation has been developed rapidly. Blood circulation is a complex system that can cause great damage to the whole body when a certain organ is diseased. In order to better understand the physiological and pathological behavior of the human cardiovascular system, it is necessary to deeply study the dynamics of blood flow in the arteries and the mechanical factors of blood flow.

Soliton phenomena exist in many fields [1-3]. Organism is a completely nonlinear complex medium, and both the blood composition and the structure of blood vessels show obvious nonlinear characteristics. The nonlinearity of blood flow has long been discovered by Womersley $[4,5]$ and McDonald [6,7], which also provides a new direction and way for people to understand the law of life movement. Since then, many scholars have begun to study the field and made great progress. Ravindran et al. [8] derived the nonlinear Schödinger equation (the terms of pseudodifferential operators) governing the modulation of periodic waves. The $\mathrm{KdV}$ equation describing blood flow is obtained by Sigeo [9], to explain the steepness of pressure waves during propagation. Hashizume [10] analyzed the propagation of pressure waves from a theoretical perspective. Liu [11] combined arterial flow as a balanced flow with periodic small pulsatile flow and analyzed the effect of vascular elasticity on blood flow. Demiray $[12,13]$ considered propagation of wave through a viscous incompressible fluid contained in a prestressed thin elastic tube. Choy [14] deduced the mathematical model of nonlinear wave modulation of artery with stenosis.

Fractional derivative theory and methods [15-18] are widely used in the study of nonequilibrium systems of 
various intermediate processes and critical phenomena in physics and mechanics, especially in nonlinear science [19-22]. Fractional differential equations are transformed in a standard differential equation by replacing the time derivative or the space derivative with the fractional derivative. Compared with integer-order differential equations, the most important advantage of fractional derivative equations is that it can better fit some natural physical processes and dynamic system processes. Moreover, the study of solving partial differential equations also has a new exploration, such as the extended tanh method [23], the exp-function method [24], the variational-iteration method $[15,25]$, the Hirota bilinear method [19], and the $\left(G^{\prime} / G\right)$-expansion method [26].

The structure of the full article is as follows: in Section 2, the Boussinesq equation with viscous term is derived by the multiscale analysis and perturbation method and used for the first time to describe blood flow. Based on the new model, we obtain the time-fractional Boussinesq equation with viscous term in Section 3. In Section 4, the approximate analytical solution of the above fraction equation and the viscous effect is discussed. Finally, the effects of fractional order, vascular radius, and blood flow velocity on stroke volume are analyzed and studied.

\section{Derivation of the Boussinesq Equation}

As we all know, the cardiovascular system is a complete closed conduit system. Thus, the blood can be considered as an incompressible non-Newtonian fluid. When dynamic equilibrium of the blood is disturbed by a pressure pulse generated by the motion of the heart, a harmonic wave type of motion will be developed in the blood. Although previous researchers have done some research about this question, the viscosity of fluid is often ignored. We consider blood vessel as cylindrical shape and adopt cylindrical coordinate system to describe the motion of blood. In this paper, we set $x$ as the central axis of a blood vessel and axial coordinate and set $r$ as the radial coordinates. Therefore, based on Womersley theory [4], basic equations of pulsatile flow in arteries are described as follows:

$$
\begin{aligned}
\rho\left(\frac{\partial u}{\partial t}+u \frac{\partial u}{\partial x}+v \frac{\partial u}{\partial r}\right)-\eta\left(\frac{\partial^{2} u}{\partial t^{2}}+\frac{1}{r} \frac{\partial u}{\partial r}+\frac{\partial^{2} u}{\partial x^{2}}\right) & =-\frac{\partial P}{\partial x}, \\
\rho\left(\frac{\partial v}{\partial t}+u \frac{\partial v}{\partial x}+v \frac{\partial v}{\partial r}\right)-\eta\left(\frac{\partial^{2} v}{\partial t^{2}}+\frac{1}{r} \frac{\partial v}{\partial r}+\frac{\partial^{2} v}{\partial x^{2}}-\frac{v}{r^{2}}\right) & =-\frac{\partial P}{\partial r}, \\
\frac{\partial u}{\partial x}+\frac{1}{r} \frac{\partial r v}{\partial r} & =0
\end{aligned}
$$

where $u$ and $v$ are the axial and radial velocities of blood, respectively; $p$ is the atmospheric pressure; $\rho$ is the blood density; and $\eta$ represents the viscosity coefficient of blood. From (3), we can define the flow function of blood which satisfies

$$
\begin{aligned}
& u=\frac{1}{r} \frac{\partial \Psi}{\partial r}, \\
& v=-\frac{1}{r} \frac{\partial \Psi}{\partial x} .
\end{aligned}
$$

Substituting (4) into (1) and (2), we can gain the vorticity equation of blood flow with viscosity term:

$$
\begin{gathered}
\rho\left(\frac{\partial}{\partial t}+\frac{1}{r} \frac{\partial \Psi}{\partial r} \frac{\partial}{\partial x}-\frac{1}{r} \frac{\partial \Psi}{\partial r}+\frac{2}{r^{2}} \frac{\partial \Psi}{\partial x}\right) D^{2} \Psi \\
-\eta\left(\frac{\partial^{2}}{\partial r^{2}}-\frac{1}{r} \frac{\partial}{\partial r}+\frac{\partial^{2}}{\partial x^{2}}\right) D^{2} \Psi=0,
\end{gathered}
$$

where $D^{2} \equiv\left(\left(\partial^{2} / \partial r^{2}\right)-(1 / r)(\partial / \partial r)+\left(\partial^{2} / \partial x^{2}\right)\right)$. Through analysis, we assume that the basic law of blood flow can be expressed by the Poiseuille flow. Therefore, the speed of blood can be expressed as

$$
\bar{u}(r)=u_{\max }\left[1-\left(\frac{r}{R}\right)^{2}\right],
$$

where $u_{\max }=\left(\Delta p R^{2} / 4 \mu \Delta L\right)$ is the maximum velocity of central axis of the blood vessel. $\Delta L$ represents the length of the blood fluid, and $\mu$ represents viscosity coefficient of blood. $\Delta p$ is the pressure difference between two ends of blood, and $R$ is the radius of the blood vessel. Next, when considering the viscosity of the vascular wall, we can get the revised form of the Poiseuille flow:

$$
\bar{u}(r)=u_{\max }\left[\frac{2 r^{2}}{R^{2}} \ln \frac{r}{R}+1-\left(\frac{r}{R}\right)^{2}\right] .
$$

We set basic flow function of blood as $\bar{\psi}$. Therefore, it meets

$$
\bar{u}=\frac{1}{r} \frac{\partial \bar{\psi}}{\partial r},
$$

where we define disturbed flow function $\varphi(r, x, t)$ which represents disturbance of blood flow. Therefore, the flow function can be expressed as

$$
\Psi(r, x, t)=\bar{\psi}(r)+\varphi(r, x, t) .
$$

And then, from (8) and (4), we can obtain

$$
\begin{aligned}
& u=\bar{u}+\frac{1}{r} \frac{\partial \varphi}{\partial r}, \\
& v=-\frac{1}{r} \frac{\partial \varphi}{\partial x} .
\end{aligned}
$$

Substituting (9) and (10) into (5) and considering that there are other effects of dissipation which can offset $(2 a \eta / \rho)$, we obtain

$$
\begin{aligned}
& \left(\frac{\partial}{\partial t}+\frac{1}{r} \frac{\partial \varphi}{r} \frac{\partial}{\partial x}-\frac{1}{r} \frac{\partial \varphi}{\partial x} \frac{\partial}{\partial r}+\frac{2}{r^{2}} \frac{\partial \varphi}{\partial x}+\bar{u} \frac{\partial}{\partial x}\right) D^{2} \varphi-a \frac{\partial \varphi}{\partial x} \\
& =\frac{\eta}{\rho}\left(D^{2} D^{2} \varphi\right),
\end{aligned}
$$


where $a=\left(4 u_{\max } / R^{2}\right)$ and $\lim _{r \longrightarrow 0} \bar{u}(r)=u_{\max }$. The above equation is a vorticity equation of disturbed flow function $\varphi$ which contains information about the effects on the heart when physiological or psychological condition changes dramatically.

In the previous studies, researchers have obtained the $\mathrm{KdV}$ equation from the Navier-Stokes equation and continuity equation of blood flow. However, with the development of nonlinear theory increasing, more equations which have stronger nonlinear properties are needed to describe problems in reality. Therefore, the Boussinesq equation model will be deduced in this paper. In order to obtain this equation, we firstly define coordinate transformations with long wave approximation and adopt the space-time transformation as follows:

$$
\begin{aligned}
X & =\varepsilon(x-c t), \\
T & =\varepsilon^{2} t, \\
r & =r,
\end{aligned}
$$

and we set

$$
\frac{\eta}{\rho}=\varepsilon^{3} Q
$$

where $\varepsilon$ is a small parameter. Substituting (12) into (11) yields

$$
\begin{gathered}
\left(\frac{\partial}{\partial T} \varepsilon^{2}+\frac{1}{r} \frac{\partial \varphi}{\partial r} \frac{\partial}{\partial X} \varepsilon-\frac{1}{r} \frac{\partial \varphi}{\partial X} \frac{\partial}{\partial r} \varepsilon+\frac{2}{r^{2}} \frac{\partial \varphi}{\partial X} \varepsilon+(\bar{u}-c) \frac{\partial}{\partial X} \varepsilon\right)\left(\frac{\partial^{2}}{\partial r^{2}}-\frac{1}{r} \frac{\partial}{\partial r}+\frac{\partial^{2}}{\partial X^{2}} \varepsilon^{2}\right) \varphi-a \frac{\partial \varphi}{\partial X} \varepsilon \\
=Q \varepsilon^{3}\left[\left(\frac{\partial^{2}}{\partial r^{2}}-\frac{1}{r} \frac{\partial}{\partial r}+\frac{\partial^{2}}{\partial X^{2}} \varepsilon^{2 \alpha}\right)\left(\frac{\partial^{2}}{\partial r^{2}}-\frac{1}{r} \frac{\partial}{\partial r}+\frac{\partial^{2}}{\partial X^{2}} \varepsilon^{2}\right) \varphi\right] .
\end{gathered}
$$
follows:

$$
\varphi=\varepsilon^{2} \varphi_{0}+\varepsilon^{3} \varphi_{1}+\varepsilon^{4} \varphi_{2}+\cdots .
$$

Due to the fact that the velocity of wave propagation in blood is much faster than that of blood flow on the axis of the blood vessel, we can obtain $\bar{u} \ll c$. Thus, substituting (15) into (14), we can yield all levels of approximation equations about $\varepsilon$ :

$$
\begin{aligned}
\varepsilon^{3}: \Gamma \varphi_{0}= & 0 \\
\varepsilon^{4}: \Gamma \varphi_{1}= & -\frac{\partial}{\partial T}\left(\frac{\partial^{2}}{\partial r^{2}}-\frac{1}{r} \frac{\partial}{\partial r}\right) \varphi_{0}, \\
\varepsilon^{5}: \Gamma \varphi_{2}= & -\frac{\partial}{\partial T}\left(\frac{\partial^{2}}{\partial r^{2}}-\frac{1}{r} \frac{\partial}{\partial r}\right) \varphi_{1}+c \frac{\partial^{3}}{\partial X^{3}} \varphi_{0} \\
& -\left(\frac{1}{r} \frac{\partial \varphi_{0}}{\partial r} \frac{\partial}{\partial X}-\frac{1}{r} \frac{\partial \varphi_{0}}{\partial X} \frac{\partial}{\partial r}+\frac{2}{r^{2}} \frac{\partial \varphi_{0}}{\partial X}\right)\left(\frac{\partial^{2}}{\partial r^{2}}-\frac{1}{r} \frac{\partial}{\partial r}\right) \varphi_{0} \\
& +Q\left[\left(\frac{\partial^{2}}{\partial r^{2}}-\frac{1}{r} \frac{\partial}{\partial r}\right)\left(\frac{\partial^{2}}{\partial r^{2}}-\frac{1}{r} \frac{\partial}{\partial r}\right) \varphi_{0}\right],
\end{aligned}
$$

where $\Gamma \equiv-c(\partial / \partial X)\left(\left(\partial^{2} / \partial r^{2}\right)-(1 / r)(\partial / \partial r)\right)-a(\partial / \partial X)$.

For $\varepsilon^{3}$, it is easy to see that $\varphi_{0}$ has a solution, and the form is $\varphi_{0}=A(X, T) \phi_{0}(r)$; then, we have

$$
-c\left(\frac{\partial^{2}}{\partial r^{2}}-\frac{1}{r} \frac{\partial}{\partial r}\right) \phi_{0}-a \phi_{0}=0 .
$$

For $\varepsilon^{4}$, by analysis, we assume $\left(\partial \varphi_{1} / \partial X\right)=$ $(\partial A / \partial T) \phi_{1}(r)$, where $\phi_{1}(r)$ satisfies the following equation:

$$
-c\left(\frac{\partial^{2}}{\partial r^{2}}-\frac{1}{r} \frac{\partial}{\partial r}\right) \phi_{1}-a \phi_{1}=\frac{a}{c} \phi_{0} .
$$

Let us consider the equation of order $\varepsilon^{5}$. By substituting $\varphi_{0}=A(X, T) \phi_{0}(r), \quad\left(\partial \varphi_{1} / \partial X\right)=(\partial A / \partial T) \phi_{1}(r), \quad(17)$, and (18) into the equation and multiplying the both sides of the order $\varepsilon^{5}$ equation by $\left(\phi_{0} / r\right)(\partial / \partial X)$, we have

$$
\begin{aligned}
\frac{\partial^{2}}{\partial X^{2}} & \frac{\phi_{0}}{r}\left(\frac{\partial^{2}}{\partial r^{2}}-\frac{1}{r} \frac{\partial}{\partial r}\right) \varphi_{2}+a \frac{\partial^{2} \varphi_{2}}{\partial X^{2}} \frac{\phi_{0}}{r} \frac{1}{c} \\
= & -\frac{\partial^{2} A}{\partial T^{2}} \frac{\phi_{0}}{r} \frac{a}{c^{2}}\left(\phi_{1}+\frac{\phi_{0}}{c}\right)-\frac{\partial^{4} A}{\partial X^{4}} \frac{\phi_{0}^{2}}{r} \\
& -\frac{a}{c^{2}} \frac{\phi_{0}^{2}}{r^{3}} \frac{\partial^{2} A^{2}}{\partial X^{2}}-Q \frac{\partial A}{\partial X} \frac{1}{c} \frac{\phi_{0}}{r}\left(\frac{\partial^{2}}{\partial r^{2}}-\frac{1}{r} \frac{\partial}{\partial r}\right)^{2} \phi_{0} .
\end{aligned}
$$

And then integrating it with respect to $r$ from 0 to $R$, we can have the following equation:

$$
\frac{\partial^{2} A}{\partial T^{2}}+a_{1} \frac{\partial^{2} A^{2}}{\partial X^{2}}+a_{2} \frac{\partial^{4} A}{\partial X^{4}}+a_{3} \frac{\partial A}{\partial X}=0,
$$

where

$$
\begin{aligned}
& a_{0}=\int_{0}^{R} \frac{\phi_{0}}{r} \frac{a}{c^{2}}\left(\phi_{1}+\frac{\phi_{0}}{c}\right) \mathrm{d} r, \\
& a_{1}=\int_{0}^{R} \frac{\left(a / c^{2}\right)\left(\phi_{0}^{2} / r^{3}\right) \mathrm{d} r}{a_{0}}, \\
& a_{2}=\int_{0}^{R} \frac{\left(\phi_{0}^{2} / r\right) \mathrm{d} r}{a_{0}}, \\
& a_{3}=Q \int_{0}^{R} \frac{\left((1 / c)\left(\phi_{0} / r\right)\right)\left(\left(\partial^{2} / \partial r^{2}\right)-(1 / r)(\partial / \partial r)\right)^{2} \phi_{0} \mathrm{~d} r}{a_{0}} .
\end{aligned}
$$


Equation (20) is a new Boussinesq equation with viscosity effect which can be used to describe the state of the disturbance flow of blood with viscous properties. Comparing with the previous $\mathrm{KdV}$ model, the above model has stronger nonlinear properties. Besides, it can be concluded that there is disturbance flow in the form of solitary waves in the arteries.

\section{Derivation of the Time-Fractional Boussinesq Equation}

In this section, we seek for the time-fractional Boussinesq equation by using the semi-inverse method and the fractional variational principle. Firstly, we use a potential function $V_{X}(X, T)$, where $A(X, T)=V_{X}(X, T)$ gives the potential equation of (20) in the following form:

$$
V_{X T T}+a_{1}\left(A^{2}\right)_{X X}+a_{2} V_{X X X X X}+a_{3} A_{X}=0 .
$$

The functional of the potential equation (22) can be represented by

$$
\begin{aligned}
J\left(V_{X}\right)= & \int_{\tilde{X}} d X \int_{\widetilde{T}} d T\left\{V _ { X } \left[c_{0} V_{X T T}+c_{1} a_{1}\left(A^{2}\right)_{X X}\right.\right. \\
& \left.\left.+c_{2} a_{2} V_{X X X X X}+c_{3} a_{3} A_{X}\right]\right\},
\end{aligned}
$$

where $c_{0}, c_{1}, c_{2}$, and $c_{3}$ are the Lagrangian multipliers which can be obtained later by taking the variation of (23). $A_{X}$ and $\left(A^{2}\right)_{X X}$ are considered as the fixed functions.

Making use of integrating (23) by parts and assuming $\left.V_{T}\right|_{T}=\left.V_{X}\right|_{\tilde{X}}=\left.V_{X X}\right|_{\tilde{X}}=\left.V_{X X X}\right|_{\tilde{X}}=0$, we can gain

$$
\begin{aligned}
J\left(V_{X}\right)= & \int_{\tilde{X}} d X \int_{\tilde{T}} d T\left\{-c_{0} V_{X T}^{2}+c_{1} a_{1} V_{X}\left(A^{2}\right)_{X X}\right. \\
& \left.+c_{2} a_{2} V_{X X X}^{2}+c_{3} a_{3} V_{X} A_{X}\right\} .
\end{aligned}
$$

Applying the variation optimum condition, we can derive the Euler equation as follows:

$$
2 c_{0} V_{X T T}+c_{1} a_{1}\left(A^{2}\right)_{X X}+2 c_{2} a_{2} V_{X X X X X}+c_{3} a_{3} A_{X}=0 .
$$

Comparing (25) with (22), we can obtain the following Lagrangian multipliers:

$$
\begin{aligned}
& c_{0}=\frac{1}{2}, \\
& c_{1}=1, \\
& c_{2}=\frac{1}{2}, \\
& c_{3}=1 .
\end{aligned}
$$

Therefore, the Lagrangian form of (20) can be given by

$$
\begin{aligned}
L\left(V_{X T}, V_{X}, V_{X X}, V_{X X X}\right)= & -\frac{1}{2} V_{X T}^{2}+a_{1} V_{X}\left(A^{2}\right)_{X X} \\
& +\frac{1}{2} a_{2} V_{X X X}^{2}+a_{3} V_{X} A_{X} .
\end{aligned}
$$

At this time, the Lagrangian form of the time-fractional Boussinesq equation can be represented as

$$
\begin{aligned}
F\left({ }_{0} D_{T}^{\beta} V_{X}, V_{X}, V_{X X}, V_{X X X}\right)= & -\frac{1}{2}\left({ }_{0} D_{T}^{\beta} V_{X}\right)^{2}+a_{1} V_{X}\left(A^{2}\right)_{X X} \\
& +\frac{1}{2} a_{2} V_{X X X}^{2}+a_{3} V_{X} A_{X},
\end{aligned}
$$

where based on the left Riemann-Liouville compression derivative, the fractional derivative ${ }_{0} D_{T}^{\beta} V_{X}$ is defined as

$$
\begin{array}{r}
{ }_{0} D_{T}^{\beta} f(T)=\frac{1}{\Gamma(1-\beta)} \frac{\mathrm{d}}{\mathrm{d} T}\left[\int_{a}^{T} \mathrm{~d} \tau(T-\tau)^{-\beta} f(\tau)\right], \\
0 \leq \beta \leq 1, T \in[a, b] .
\end{array}
$$

Therefore, the time-fractional form of (20) can be expressed by

$$
J\left(V_{X}\right)=\int_{\widetilde{X}} \mathrm{~d} X \int_{\widetilde{T}} \mathrm{~d} T F\left({ }_{0} D_{T}^{\beta} V_{X}, V_{X}, V_{X X}, V_{X X X}\right) .
$$

Thus, the variation of functional (30) can be obtained:

$$
\begin{aligned}
\delta J\left(V_{X}\right)= & \int_{\widetilde{X}} \mathrm{~d} X \int_{\widetilde{T}} \mathrm{~d} T\left\{\frac{\partial F}{\partial_{0} D_{T}^{\beta} V_{X}} \delta\left({ }_{0} D_{T}^{\beta} V_{X}\right)+\frac{\partial F}{\partial V_{X}} \delta V_{X}\right. \\
& \left.+\frac{\partial F}{\partial V_{X X}} \delta V_{X X}+\frac{\partial F}{\partial V_{X X X}} \delta V_{X X X}\right\} .
\end{aligned}
$$

As we all know, the fractional integration by parts is obtained by the following rule:

$$
\begin{array}{r}
\int_{b}^{a} \mathrm{~d} T\left\{f(T)_{a} D_{T}^{\beta} g(T)\right\}=\int_{a}^{b} \mathrm{~d} T\left\{g(T)_{T} D_{b}^{\beta} f(T)\right\}, \\
f(T), g(T) \in[a, b],
\end{array}
$$

based on the right Riemann-Liouville fractional derivative, ${ }_{0} D_{T}^{\beta} f(T)$ is obtained by

$$
\begin{array}{r}
{ }_{T} D_{T}^{\beta} f(T)=\frac{-1}{\Gamma(1-\beta)} \frac{\mathrm{d}}{\mathrm{d} T}\left[\int_{T}^{b} \mathrm{~d} \tau(\tau-T)^{-\beta} f(\tau)\right], \\
0 \leq \beta \leq 1, T \in[a, b] .
\end{array}
$$

Using (31) and (32), we get

$$
\begin{aligned}
\delta J\left(V_{X}\right)= & \int_{\tilde{X}} \mathrm{~d} X \int_{\widetilde{T}} \mathrm{~d} T\left\{{ }_{T} D_{b}^{\beta}\left(\frac{\partial F}{\partial_{0} D_{T}^{\beta} V_{X}}\right)+\frac{\partial F}{\partial V_{X}}\right. \\
& \left.-\frac{\partial}{\partial X}\left(\frac{\partial F}{\partial V_{X X}}\right)+\frac{\partial^{2}}{\partial X^{2}}\left(\frac{\partial F}{\partial V_{X X X}}\right)\right\} \delta V_{X} .
\end{aligned}
$$

By using variation principle, when $\delta J(V)=0$, we obtain the optimization of the variation of functional. And then, we yield the Euler-Lagrange equation of (20) as the following equation: 
$-{ }_{0} D_{T}^{\beta}\left(\frac{\partial F}{\partial_{0} D_{T}^{\beta} V_{X}}\right)+\frac{\partial F}{\partial V_{X}}-\frac{\partial}{\partial X}\left(\frac{\partial F}{\partial V_{X X}}\right)+\frac{\partial^{2}}{\partial X^{2}}\left(\frac{\partial F}{\partial V_{X X X}}\right)=0$.

Substituting (28) into (35) and letting ${ }_{0} D_{T}^{\beta} V_{X}=A(X, T)$, (35) is turned into the following form:

$$
{ }_{0} D_{T}^{2 \beta} A(X, T)+a_{1}\left(A^{2}\right){ }_{X X}+a_{2} A_{X X X X}+a_{3} A_{X}=0 .
$$

Equation (36) is a new model, which is gained by the time-fractional method. Therefore, it is named as timefractional Boussinesq equation.

\section{Solution of the Time-Fractional Boussinesq Equation}

In the previous section, a new time-fractional Boussinesq equation had been gained. It can be used to describe the flow characteristics of blood with viscous properties. Due to this reason, we decide to yield the solution of the time-fractional Boussinesq equation to further characterize the flow state of blood.

Let we define the fractional complex transform as follows:

$$
\tau=\frac{T^{\alpha}}{\Gamma(1+\alpha)},
$$

where $\alpha$ is the unknown constant. And then, we can yield the fractional derivatives into classical derivatives with (37):

$$
\frac{\partial^{\alpha} A}{\partial T^{\alpha}}=\frac{\partial^{2} A}{\partial \tau^{2}} .
$$

It is obvious that (36) can be deduced to

$$
\frac{\partial^{2} A}{\partial \tau^{2}}+a_{1}\left(A^{2}\right)_{X X}+a_{2} A_{X X X X}+a_{3} A_{X}=0 .
$$

Next, we consider to gain the solution of (39). Due to the fact that $a_{3}$ describes viscous properties of blood, we set $\mu=$ $a_{3}$ as a small parameter. Assuming that $A_{0}=A_{0}\left(X, \mu^{(1 / 2)} \tau\right)$ and considering to get the properties of (39), two time scales are defined as follows:

$$
\begin{aligned}
& \zeta=\tau, \\
& \widetilde{\eta}=\mu^{(1 / 2)} \tau,
\end{aligned}
$$

and $A$ is expanded as

$$
A(X, \tau)=A_{1}(X, \zeta, \widetilde{\eta})+\mu A_{2}(X, \zeta, \widetilde{\eta}) .
$$

Thus, we obtain all levels of approximation equations about $\mu$ :

$$
\begin{aligned}
& \mu^{0}: \frac{\partial^{2} A_{1}}{\partial \zeta^{2}}+a_{1} \frac{\partial^{2} A_{1}^{2}}{\partial X^{2}}+a_{2} \frac{\partial^{4} A_{1}}{\partial X^{4}}=0, \\
& \mu^{1}: \frac{\partial^{2} A_{2}}{\partial \zeta^{2}}+a_{1} \frac{\partial^{2}\left(A_{1} A_{2}\right)}{\partial X^{2}}+a_{2} \frac{\partial^{4} A_{2}}{\partial X^{4}}=-\frac{\partial^{2} A_{1}}{\partial \widetilde{\eta}^{2}}-\frac{\partial A_{1}}{\partial X} .
\end{aligned}
$$

For $\mu^{0}$, it is obvious that the solution of the equation can be expressed as

$$
A_{1}(X, \zeta, \widetilde{\eta})=A_{0} \operatorname{sech}^{2}\left[\left(\frac{a_{1} A_{0}}{6 a_{2}}\right)^{(1 / 2)}(X-\nu \zeta)+\widetilde{c}\right],
$$

where $v=\left(-\left(2 a_{1} A_{0} / 3\right)\right)^{(1 / 2)}$ and $A_{0}$ represents the maximum amplitude at initial moment. Then, for $\mu^{1}$, we use the equation to determine the form of $A_{0}$. Assuming that

$$
\begin{aligned}
A_{2} & =B(I), \\
I & =X+\zeta .
\end{aligned}
$$

By substituting (44) into the order one of $\mu$ equation from (42), the following equation is obtained:

$$
\frac{\partial^{2} B}{\partial I^{2}}+a_{1} \frac{\partial^{2}}{\partial I^{2}}\left(A_{1} B\right)+a_{2} \frac{\partial^{4} B}{\partial I^{4}}=M\left(A_{1}\right),
$$

where $M\left(A_{1}\right)=-\left(\partial^{2} A_{1} / \partial \widetilde{\eta}^{2}\right)-\left(\partial A_{1} / \partial X\right)$. In order to describe the structure of $A_{2}$, we consider to choose proper function $G$ which should be orthogonal to $M\left(A_{1}\right)$ as

$$
\int_{-\infty}^{+\infty} G(I) M\left(A_{1}\right) \mathrm{d} I=0 .
$$

Multiplying both sides of (45) by $G$, integrating them by parts and using $\left.B\right|_{I= \pm \infty}=0$ and $\left.(\mathrm{d} B / \mathrm{d} I)\right|_{I= \pm \infty}=0$, we can see that $G(I)$ satisfies

$$
\frac{\mathrm{d}^{2} G}{\mathrm{~d} I^{2}}+a_{1} A_{1} \frac{\mathrm{d}^{2} G}{\mathrm{~d} I^{2}}+a_{2} \frac{\mathrm{d}^{4} G}{\mathrm{~d} I^{4}}=0 .
$$

By assuming $G( \pm \infty)=0$, the solution of (47) can be obtained as

$$
G=A_{0} \sec h^{2}\left[\left(\frac{a_{1} A_{0}}{6 a_{2}}\right)^{(1 / 2)} I+\tilde{c}\right] .
$$

Substituting (48) into (46) and using (45), we obtain

$$
A_{0}=e^{-\sqrt{\mu} \tau-X} .
$$

Therefore, we obtain the approximate solution of (39) as follows:

$$
A(X, \zeta, \widetilde{\eta})=A_{0} \sec h^{2}\left[\left(\frac{a_{1} A_{0}}{6 a_{2}}\right)^{(1 / 2)}(X-\nu \zeta)+\widetilde{c}\right],
$$

where $A_{0}\left(X, \mu^{(1 / 2)} \tau\right)=e^{-\sqrt{\mu} \tau-X}$. The above equation is the solution of (39) and can be used to describe the flow state of blood more specifically.

Finally, the solution of the time-fractional Boussinesq equation is

$$
\begin{aligned}
A(X, T)= & \exp \left(\frac{-\sqrt{\mu} T^{\alpha}}{\Gamma(1+\alpha)}-X\right) \\
& \cdot \sec h^{2}\left[\left(\frac{a_{1}}{6 a_{2}} \exp \left(\frac{-\sqrt{\mu} T^{\alpha}}{\Gamma(1+\alpha)}-X\right)\right)^{(1 / 2)}\right. \\
& \left.\cdot\left(X-v \frac{T^{\alpha}}{\Gamma(1+\alpha)}\right)+\widetilde{c}\right],
\end{aligned}
$$


where $\nu=\left(-\left(2 a_{1} / 3\right) \exp \left(\left(-\sqrt{\mu} T^{\alpha} / \Gamma(1+\alpha)\right)-X\right)\right)^{(1 / 2)}$.

In order to study the influence of blood viscosity and fractional order on the evolution of blood flow, we change the value of $\mu, \alpha, X$, and $T$ to get related results. From Figure 1, we can see that when viscosity parameter $\mu=0$, the amplitude of solitary waves is largest; at the same time, the width of solitary waves is the smallest. With the decrease of the amplitude, the width increases gradually. Figure 2 depicts the evolution of solitary waves in blood under different viscosity coefficient $\mu$. It is evident that amplitude of the solitary waves becomes smaller with time $T$ from Figure 2(a), while the width of the solitary waves becomes larger with time $T$. And we can know that the viscosity effect leads to the amplitude of the solitary waves to decrease and the width of the solitary waves to increase. In addition, in contrast with Figure 2(b), we find with the increasing viscosity coefficient $\mu$, the rate of amplitude reduction has been accelerated and the direction of the wave changes from the positive direction of $X$ to the negative direction of it. Figure 3 shows the solitary waves under different fractional order $\alpha$. We find the peak value of amplitude is not linear with $\alpha$, and there is a minimum of the peak with the corresponding critical value of $\alpha$. According to the critical value, the peak value of amplitude increases firstly and then decreases.

\section{Analysis and Discussion}

In this section, we consider to calculate the value which can represent the state of blood by using the results from the previous sections. First, we search for the solution of (17) and (18). Taking $\phi_{0}(r)=\phi_{0}((\sqrt{c / a}) z)=z w(z)$ and substituting them into (17) yield

$$
z^{2} \frac{\mathrm{d}^{2} w}{\mathrm{~d} z^{2}}+z \frac{\mathrm{d} w}{\mathrm{~d} z}+\left(z^{2}-1\right)=0
$$

And the solution of (52) is expressed as

$$
w(z)=C_{1} J_{1}(z)+C_{2} N_{1}(z)
$$

where $C_{1}$ and $C_{2}$ are the constants and $J_{1}(z)$ and $N_{1}(z)$ are expressed as

$$
\begin{aligned}
J_{1}(z) & =\sum_{m=1}^{\infty} \frac{(-1)^{m}}{m ! \Gamma(m+2)}\left(\frac{z}{2}\right)^{2 m+1}, \\
N_{1}(z) & =\frac{J_{1}(z) \cos (\pi)-J_{-1}(z)}{\sin (\pi)} .
\end{aligned}
$$

Thus, the solution of (17) can be described as

$$
\phi_{0}(r)=C_{1} r \sqrt{\frac{a}{c}} J_{1}\left(r \sqrt{\frac{a}{c}}\right) .
$$

Similarly, by substituting (55) into (18), we obtain

$$
\phi_{1}(r)=C_{3} r \sqrt{\frac{a}{c}} J_{1}\left(r \sqrt{\frac{a}{c}}\right)
$$

where $C_{3}$ is a constant. We take the physiological parameters of the human body as follows:

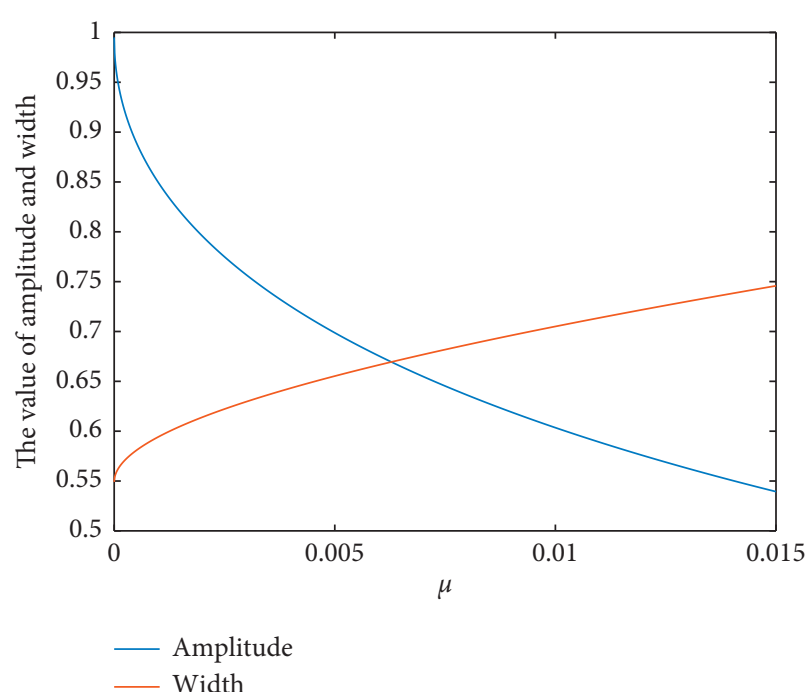

FIgURE 1: Variation of amplitude and width according to the viscosity coefficient $\mu\left(\alpha=1, T=5, X=0.005, a_{2}=-1, a_{1}=-20\right.$, $\tilde{c}=0$ ).

$$
\begin{aligned}
R & =1.25 \times 10^{-2} \mathrm{~m}, \\
u_{\max } & =0.4 \mathrm{~m} / \mathrm{s}, \\
L & =6.3 \times 10^{-2} \mathrm{~m}, \\
c & =5.5 \mathrm{~m} / \mathrm{s} .
\end{aligned}
$$

And set $Q=-0.01, C_{1}=0.1$, and $C_{3}=-500$. Therefore, by using (21), we obtain the coefficients of (36) as follows:

$$
\begin{aligned}
& a_{0}=-8.52776 \times 10^{-1}, \\
& a_{1}=-2.5917, \\
& a_{2}=-5.9084 \times 10^{-7}, \\
& a_{3}=3.7 \times 10^{-3} .
\end{aligned}
$$

Due to that the basic flow in a human artery can be assumed to be the Poiseuille flow, the stream function can be expressed as

$$
\bar{\psi}=u_{\max }\left(\frac{r^{2}}{2}-\frac{r^{4}}{4 R^{2}}\right) .
$$

So the stream function can be obtained as follows:

$$
\begin{aligned}
\Psi= & u_{\max }\left(\frac{r^{2}}{2}-\frac{r^{4}}{4 R^{2}}\right)+\varepsilon^{2} \exp \left(\frac{-\sqrt{\mu} T^{\alpha}}{\Gamma(1+\alpha)}-X\right) \\
& \cdot \sec h^{2}\left[\left(\frac{a_{1}}{6 a_{2}} \exp \left(\frac{-\sqrt{\mu} T^{\alpha}}{\Gamma(1+\alpha)}-X\right)\right)^{(1 / 2)}\right. \\
& \left.\cdot\left(X-v \frac{T^{\alpha}}{\Gamma(1+\alpha)}\right)+\widetilde{c}\right] C_{1} r \sqrt{\frac{a}{c}} J_{1}\left(r \sqrt{\frac{a}{c}}\right),
\end{aligned}
$$

where $X=\varepsilon(x-c t)$ and $T=\varepsilon^{2} t$ following (12).

Because in the cylindrical coordinate system, the difference of the flow function values of any two points in the 


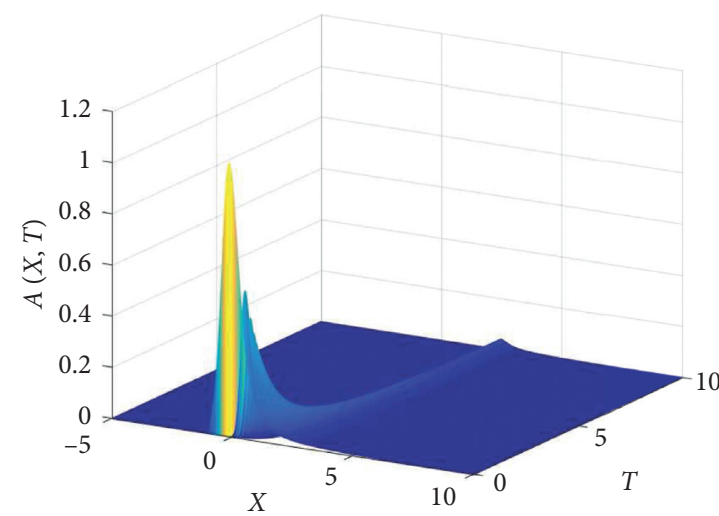

(a)

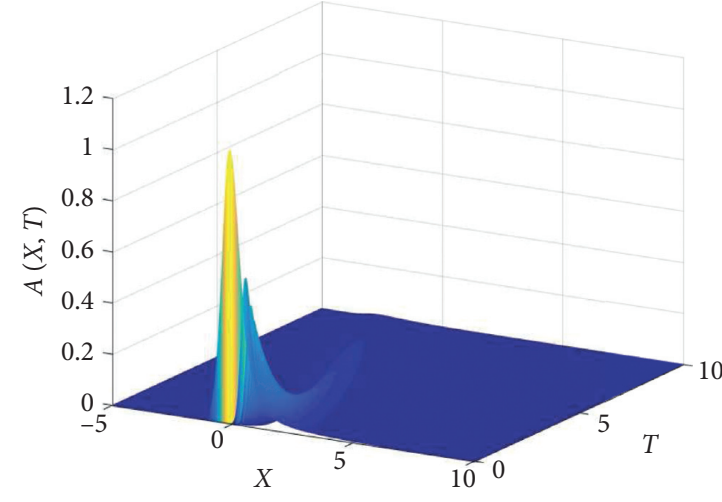

(b)

FIGURE 2: The evolution of solitary waves in blood with viscosity: (a) $\alpha=1, a_{2}=-1, a_{1}=-20, \tilde{c}=0, \mu=0.005$; (b) $\alpha=1, a_{2}=-1, a_{1}=-20$, $\widetilde{c}=0, \mu=0.45$.

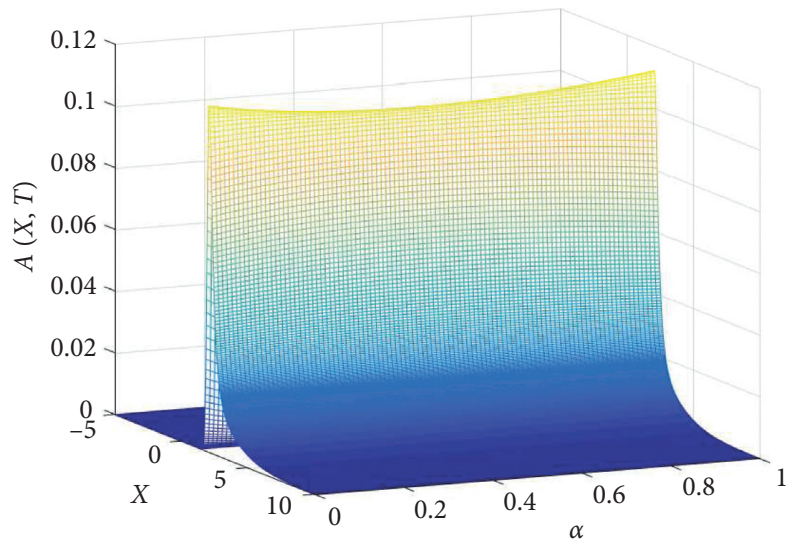

Figure 3: The evolution of solitary waves with different numbers of fractional order $\alpha\left(T=1, a_{2}=-1, a_{1}=-20, \tilde{c}=0, \mu=0.005\right)$.

plane passing through the symmetry axis multiplied by $2 \pi$ is equal to the value, which is the flow rate of the rotating surface formed by the rotation of any curve passing through these two points around the symmetric axis, we decide to calculate blood flow by using the values of the outlet and entrance of blood vessels. We take the flow function of the central position of the vessel at the entrance of the aorta as $\Psi_{1}$, where $X=0$ and $r=0$, and take the flow function at the junction of heart and aorta as $\Psi_{1}$, where $x=0$ and $r=R$. Thus, we obtain

$$
\begin{aligned}
\Psi_{1}= & 0 \\
\Psi_{2}= & \frac{1}{4} u_{\max } R^{2}+\varepsilon^{2} \exp \left(\frac{-\sqrt{\mu}\left(\varepsilon^{2} t\right)^{\alpha}}{\Gamma(1+\alpha)}+\varepsilon c t\right) C_{1} r \sqrt{\frac{a}{c}} J_{1}\left(r \sqrt{\frac{a}{c}}\right) \\
& \cdot \sec h^{2}\left[\left(\frac{a_{1}}{6 a_{2}} \exp \left(\frac{-\sqrt{\mu}\left(\varepsilon^{2} t\right)^{\alpha}}{\Gamma(1+\alpha)}+\varepsilon c t\right)\right)^{(1 / 2)}\left(-\varepsilon c t-\left(-\frac{2 a_{1}}{3} \exp \left(\frac{-\sqrt{\mu}\left(\varepsilon^{2} t\right)^{\alpha}}{\Gamma(1+\alpha)}+\varepsilon c t\right)\right)^{(1 / 2)} \frac{\left(\varepsilon^{2} t\right)^{\alpha}}{\Gamma(1+\alpha)}\right)+\widetilde{c}\right] .
\end{aligned}
$$




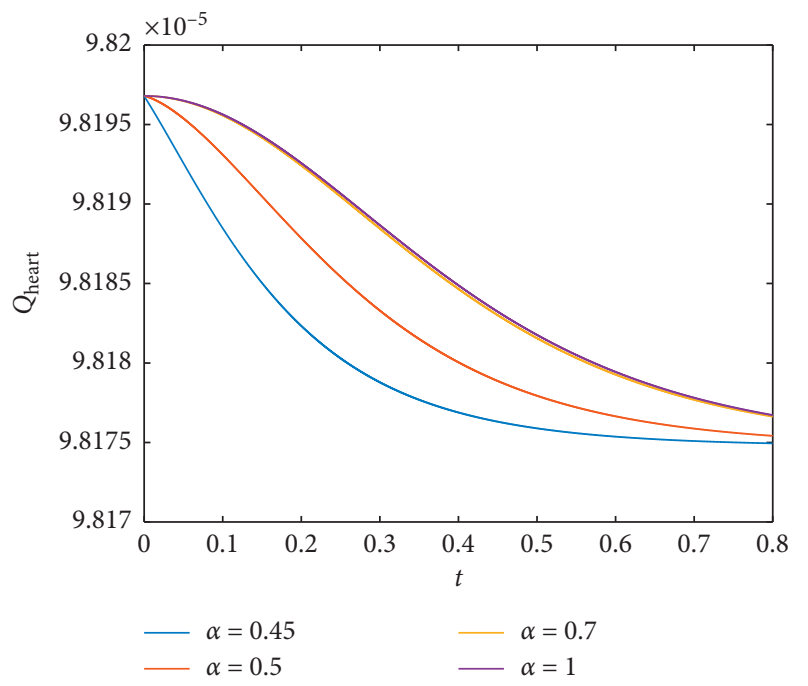

Figure 4: The blood flow volume of cardiac outlet at the different values of time and $\alpha(\widetilde{c}=0)$.

Thus, the blood flow at the outlet of the heart can be expressed as

$$
\begin{aligned}
Q_{\text {heart }}= & \frac{1}{2} \pi u_{\max } R^{2}+2 \pi \varepsilon^{2} \exp \left(\frac{-\sqrt{\mu}\left(\varepsilon^{2} t\right)^{\alpha}}{\Gamma(1+\alpha)}+\varepsilon c t\right) C_{1} R \sqrt{\frac{a}{c}} J_{1}\left(R \sqrt{\frac{a}{c}}\right) \\
& \cdot \sec h^{2}\left[\left(\frac{a_{1}}{6 a_{2}} \exp \left(\frac{-\sqrt{\mu}\left(\varepsilon^{2} t\right)^{\alpha}}{\Gamma(1+\alpha)}+\varepsilon c t\right)\right)^{(1 / 2)}\left(-\varepsilon c t+\left(\frac{2 a_{1}}{3} \exp \left(\frac{-\sqrt{\mu}\left(\varepsilon^{2} t\right)^{\alpha}}{\Gamma(1+\alpha)}+\varepsilon c t\right)\right)^{(1 / 2)} \frac{\left(\varepsilon^{2} t\right)^{\alpha}}{\Gamma(1+\alpha)}\right)+\widetilde{c}\right] .
\end{aligned}
$$

In a cardiac cycle, the time-dependent image of the blood flow volume of the cardiac outlet is shown in Figure 4.

From Figure 4, we can see that, when $\alpha$ is smaller, this model can be used to describe people with less blood flow or faster blood flow conduction. And when $\alpha$ is large, this model can be used to describe people with slow blood flow in the body, or blood output from the heart is not easy to circulate throughout the body. These people may have lesions in some parts of the blood vessels, causing obstruction to blood flow. Or they are in good physical condition, but they are at high risk of cardiovascular and cerebrovascular diseases. Furthermore, we can find the appropriate value of $\alpha$ to describe different populations by combining the actual monitoring data.

As we all know, stroke volume of healthy people is basically stable. When stroke volume is not stable for a long time, we can infer that there may be something wrong with that person's cardiovascular system. If the amount of stroke volume is smaller, it will lead to insufficient oxygen supply to other tissues and organs. On the contrary, when stroke volume is too large, it will increase the load of human blood vessels, thus causing some cardiovascular and cerebrovascular diseases. According to the above introduction, we can find that stroke volume in each cycle of the heart is an important index which can reflect the health status of human cardiovascular system. Thus, we give the description of the stroke volume as follows:

$$
V=\int_{0}^{\widetilde{T}} Q_{\text {heart }} \mathrm{d} t
$$

where $\widetilde{T}$ represents the pulsatile cycle of the heart. By calculating, with $u_{\max }=0.4$ and $R=1.25 \times 10^{-2}$, we obtain the stroke volume in each cycle of the heart as $78.54 \mathrm{ml}$ which is close to the theoretical calculation value $78.5 \mathrm{ml}$. Therefore, this model can well reflect the actual blood flow of the human body.

Considering that there is always a certain degree of difference in genetic characteristics of different people, it is easy to misjudge the disease condition if we use a unified health standard to measure the population with different characteristics. Thus, we consider to yield several different standards which can be used to measure the health of different groups of people by adjusting the value of fractional order $\alpha$. Figure 5 shows that the stroke volume increases with the increase of $\alpha$. $V$ has a larger growth rate in the range of $\alpha$ value from 0.4 to 0.8 , while it grows slowly in other value ranges. Thus, we can consider selecting the appropriate value of $\alpha$ from 0.4 to 0.8 with the actual test data. 


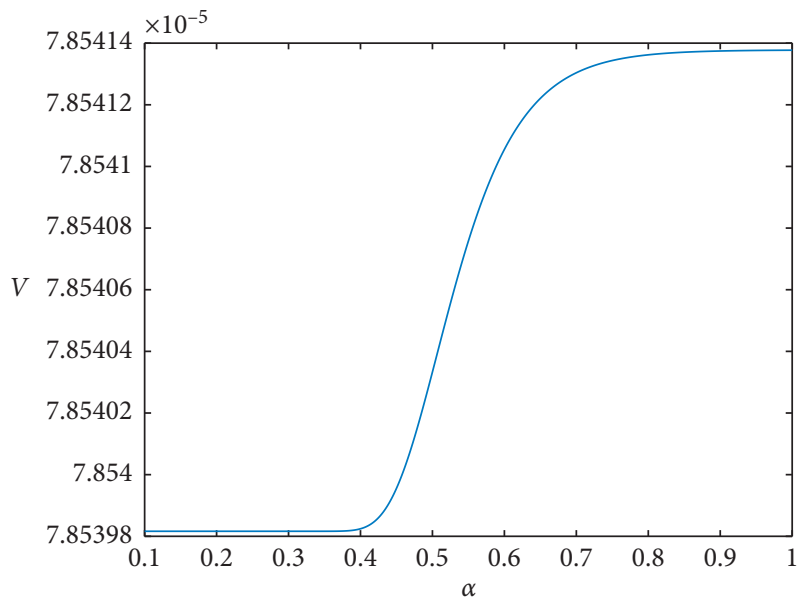

FIGURE 5: Relationship between fractional order and stroke volume $\left(R=1.25 \times 10^{-2}, u_{\max }=0.4, \widetilde{c}=0\right)$.

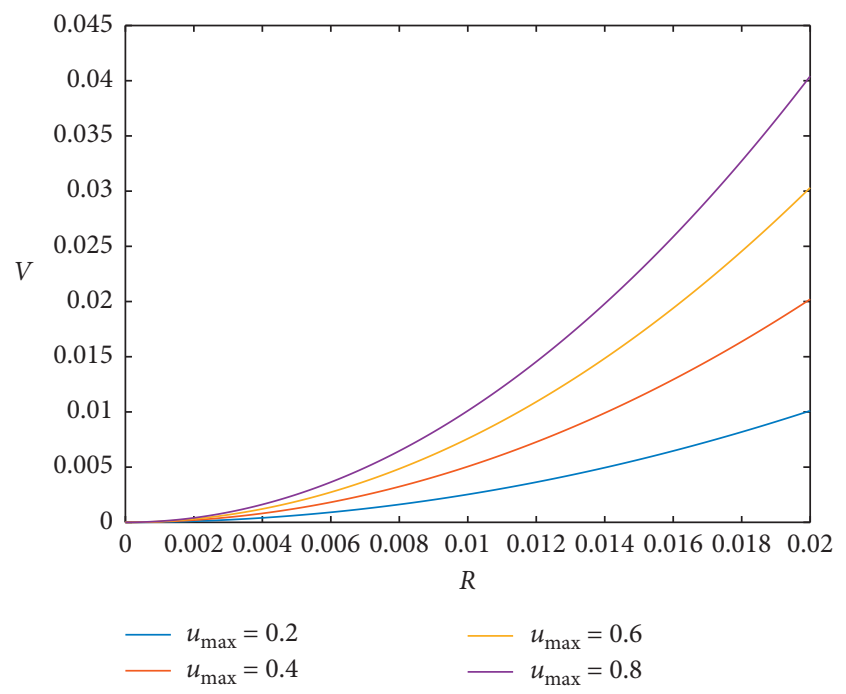

FIGURE 6: Relationship between blood vessel radius and stroke volume $(\alpha=0.5, \widetilde{c}=0)$.

Because the radius of the blood vessel can influence stroke volume, we consider changing the value of $R$ to get several results as shown in Figure 6.

In Figure 6, the values of $\alpha$ from bottom to top are 0.2, $0.4,0.6$, and 0.8 . We can find that under the condition of the same vessel radius, stroke volume increases with the increase of the maximum velocity of blood vessel center. In addition, under the same $u_{\max }$, the value of $V$ is also related to the size of the vascular radius. As the vessel radius increases, stroke volume increases as well.

\section{Conclusion}

In this paper, we derive time-fractional Boussinesq equation with viscous term to describe blood flow and discuss the effects of fractional order and viscosity on blood flow volume. This indicates that local hemodynamic factors play an important role in the occurrence and development of cardiovascular disease. Therefore, it is of great significance to study the changes of blood flow in blood vessels to reveal the pathogenesis of major vascular diseases.

\section{Data Availability}

No data were used to support the findings of the study.

\section{Conflicts of Interest}

The authors declare that there are no conflicts of interest regarding the publication of this paper.

\section{Acknowledgments}

This work was jointly sponsored by the National Key Research and Development Program of China (2017YFC1502102) and National Natural Science Foundation of China (41675102).

\section{References}

[1] Q. Liu, R. Zhang, L. Yang, and J. Song, "A new model equation for nonlinear Rossby waves and some of its solutions," Physics Letters A, vol. 383, no. 6, pp. 514-525, 2019.

[2] R. Zhang, L. Yang, Q. Liu, and X. Yin, "Dynamics of nonlinear Rossby waves in zonally varying flow with spatial-temporal varying topography," Applied Mathematics and Computation, vol. 346, pp. 666-679, 2019.

[3] M. H. Gao, Y. F. Zhang, J. F. Wang, and H. W. Yang, "A new model for describing the gravity solitary wave in the atmosphere," Mathematical Modeling and its Applications, vol. 4, pp. 10-16, 2015.

[4] J. R. Womersley, "XXIV. Oscillatory motion of a viscous liquid in a thin-walled elastic tube-I: the linear approximation for long waves," The London, Edinburgh, and Dublin Philosophical Magazine and Journal of Science, vol. 46, no. 373, pp. 199-221, 1955.

[5] J. R. Womersley, "Oscillatory flow in arteries: the constrained elastic tube as a model of arterial flow and pulse transmission," Physics in Medicine and Biology, vol. 2, no. 2, pp. 178-187, 1957.

[6] D. A. McDonald, "The velocity of blood flow in the rabbit aorta studied with high-speed cinematography," The Journal of Physiology, vol. 118, no. 3, pp. 328-339, 1952.

[7] D. A. McDonald, "The relation of pulsatile pressure to flow in arteries," The Journal of Physiology, vol. 127, no. 3, pp. 533-552, 1955.

[8] R. Ravindran and P. Prasad, "A mathematical analysis of nonlinear waves in a fluid filled visco-elastic tube," Acta Mechanica, vol. 31, no. 3, pp. 253-280, 1979.

[9] Y. Sigeo, "Solitary waves in large blood vessels," Journal of the Physical Society of Japan, vol. 56, no. 2, pp. 506-520, 1987.

[10] Y. Hashizume, "Nonlinear pressure waves in a fluid-filled elastic tube," Journal of the Physical Society of Japan, vol. 54, no. 9, pp. 3305-3312, 1985.

[11] Z. R. Liu, G. Xu, Y. Chen, Z.-Z. Teng, and K.-R. Qin, "An analysis model of pulsatile blood flow in arteries," Applied Mathematics and Mechanics, vol. 24, no. 2, pp. 230-240, 2003.

[12] H. Demiray, "Wave propagation through a viscous fluid contained in a prestressed thin elastic tube," International Journal of Engineering Science, vol. 30, no. 11, pp. 1607-1620, 1992. 
[13] H. Demiray, "Weakly nonlinear waves in a fluid with variable viscosity contained in a prestressed thin elastic tube," Chaos, Solitons \& Fractals, vol. 36, no. 2, pp. 196-202, 2008.

[14] Y. Y. Choy, K. G. Tay, and C. T. Ong, "Modulation of nonlinear waves in an inviscid fluid (blood) contained in a stenosed artery," Applied Mathematical Sciences, vol. 7, pp. 5003-5012, 2013.

[15] S. A. El-Wakil, E. M. Abulwafa, M. A. Zahran, and A. A. Mahmoud, "Time-fractional KdV equation: formulation and solution using variational methods," Nonlinear Dynamics, vol. 65, no. 1-2, pp. 55-63, 2011.

[16] F. Riewe, "Mechanics with fractional derivatives," Physical Review E, vol. 55, no. 3, pp. 3582-3592, 1997.

[17] O. P. Agrawal, "Formulation of Euler-Lagrange equations for fractional variational problems," Journal of Mathematical Analysis and Applications, vol. 272, no. 1, pp. 368-379, 2002.

[18] O. P. Agrawal, "A general formulation and solution scheme for fractional optimal control problems," Nonlinear Dynamics, vol. 38, no. 1-4, pp. 323-337, 2004.

[19] J. C. Sun, Z. G. Zhang, H. H. Dong, and H. W. Yang, "Fractional order model and lump solution in dusty plasma," Acta Physica Sinica, vol. 68, no. 21, Article ID 210201, 2019.

[20] S. Y. Lukashchuk, "Conservation laws for time-fractional subdiffusion and diffusion-wave equations," Nonlinear Dynamics, vol. 80, no. 1-2, pp. 791-802, 2015.

[21] Q. S. Liu and L. G. Chen, "Time-Space fractional model for complex cylindrical ion-acoustic waves in ultrarelativistic plasmas," Complexity, vol. 2020, Article ID 9075823, 16 pages, 2020.

[22] Z. B. Bai, "The introduction to the fractional differential equation boundary value problems," Mathematical Modeling and its Applications, vol. 6, pp. 1-10, 2017.

[23] K. R. Raslan, K. K. Ali, and M. A. Shallal, "The modified extended tanh method with the Riccati equation for solving the space-time fractional EW and MEW equations," Chaos, Solitons \& Fractals, vol. 103, pp. 404-409, 2017.

[24] O. Guner, A. Bekir, and H. Bilgil, "A note on exp-function method combined with complex transform method applied to fractional differential equations," Advances in Nonlinear Analysis, vol. 4, no. 3, pp. 201-208, 2015.

[25] S. Momani, Z. Odibat, and A. Alawneh, "Variational iteration method for solving the space-and time-fractional $\mathrm{KdV}$ equation," Numerical Methods for Partial Differential Equations, vol. 24, no. 1, pp. 262-271, 2008.

[26] N. Shang and B. Zheng, "Exact solutions for three fractional partial differential equations by the $\left(G^{\prime} / G\right)$ method," International Journal of Applied Mathematics, vol. 43, no. 3, pp. 1-6, 2013. 